\section{Optical coherence tomography findings in spinocerebellar ataxia-3}

G Alvarez', A Rey ${ }^{1}$, FB Sanchez-Dalmau', E Muñoz ${ }^{2}$, J Ríos ${ }^{3}$ and A Adán ${ }^{1}$

\begin{abstract}
${ }^{1}$ Department of
Ophthalmology, Hospital Clinic de Barcelona, Calle Sabino de Arana, Barcelona, Spain
\end{abstract}

${ }^{2}$ Department of Neurology, Hospital Clínic i Provincial de Barcelona, Calle Villarroel, Barcelona, Spain

${ }^{3}$ Laboratory of Biostatistics and Epidemiology, Hospital Clínic de Barcelona, calle Villarroel, Barcelona, Spain

Correspondence:

G Alvarez, Department of Ophthalmology, Hospital Clinic de Barcelona, Calle Sabino de Arana 08028, Barcelona, Spain

Tel: + 34 932275667;

Fax: + 34932275662

E-mail: guifre.alvpar@ gmail.com

Received: 25 February 2013 Accepted in revised form:

8 August 2013

Published online:

13 September 2013

\begin{abstract}
Purpose To report optical coherence tomography (OCT) findings in order to detect subclinical alterations of the afferent visual pathways in spinocerebellar ataxia 3 (SCA-3). Patients and methods Nine genetically confirmed patients (18 eyes) were evaluated with a complete ophthalmologic examination including visual acuity, colour vision, visual field test, and retinal nerve fibre layer (RNFL) and macular thickness with OCT Cirrus HD. A neurological examination was performed and the Scale for the Assessment and Rating of Ataxia (SARA score) was determined in all patients.

Results The mean RNFL thickness was 77.39 microns, standard deviation (SD) was \pm 5.93 . In 15 eyes $(83.33 \%)$, the mean RNFL thickness was lower than the population average considering age and sex. In 10 cases, there was a reduction of the RNFL thickness in the superior sector, eight in the inferior and four in the nasal. Temporal sector RNFL thickness was preserved in all eyes. RNFL thickness was inversely correlated to SARA score $(r=-0.64, P=0.012)$. The mean macular thickness was 252.61 microns, $\mathrm{SD} \pm \mathbf{2 2 . 8 0}$, being inferior respecting average population in only two eyes $\mathbf{( 1 1 . 1 1 \% )}$. In four patients, (eight eyes) OCT studies were performed during a mean follow-up of $\mathbf{1 4 . 2 5}$ months, and in five eyes $(62.50 \%)$ there was a mild trend to a RNFL thickness decrease in this period.

Conclusion A mild and progressive decrease in RNFL thickness can be observed in SCA-3 patients. A negative correlation exists between an anamic marker (RNFL thickness) and a clinical severity scale (SARA score); thus, RNFL thickness could be considered as a promising biomarker of the disease.
\end{abstract}

Eye (2013) 27, 1376-1381; doi:10.1038/eye.2013.201; published online 13 September 2013

Keywords: spinocerebellar ataxia 3; optical coherence tomography; retinal nerve fibre layer thickness; macular thickness; SARA score

\section{Introduction}

The autosomal dominant spinocerebellar ataxias (SCA) are a heterogeneous group of dominantly inherited neurological disorders characterised by progressive ataxia that results from the degeneration of the cerebellum and its afferent and efferent connections. ${ }^{1}$ In most forms, there is a clinical and neuropathological evidence of additional involvement of the brainstem, basal ganglia, spinal cord, and the peripheral nervous system. Disturbances of coordinated eye movements are invariable and often disabling part of the clinical syndrome. The neural mechanisms that govern the control of the eye movements reside in the brainstem, cerebellum, and the frontal, parietal, and occipital lobes of the cerebral cortex. Most of the structures concerned with eye movements are involved in the degenerative process underlying SCA. In addition, in SCA-7 a cone-rod degeneration is associated, preceding, accompanying, or following the onset of ataxic symptoms. ${ }^{2}$ On the other hand in some recessive ataxias such as Friedreichs ataxia (FA) an afferent visual pathways involvement has been ascertained. In addition, Fortuna et $a l^{3}$ detected subclinically optic neuropathy with optical coherence tomography (OCT), demonstrating a variably reduction of the retinal nerve fibre layer (RNFL) in all their patients affected of FA. Moreover, in some neurodegenerative diseases such as Parkinson's 
and Alzheimer's disease a thinning of the RNFL has been found. ${ }^{4,5}$

SCA-3, also known as Machado-Joseph disease (MJD), is the most common form of SCA in Spain, although SCA is a very infrequent disease (the estimated prevalence is about 1-4/100.000). MJD usually begins as a progressive ataxia accompanied by lid retraction and infrequent blinking, ophthalmoparesis, and impaired speech and swallowing. Neuropathological findings include widespread degeneration of cerebellar afferent and efferent pathways, pontine and dentate nuclei, and the cell bodies of substantia nigra, subthalamic nucleus, globus pallidus, cranial motor nerve nuclei, and anterior horns.

Regarding the visual system affection in one form of SCA and the subclinical optic neuropathy detected in some other forms of ataxia and other neurodegenerative diseases, we studied the clinical and OCT findings in a group of patients with MJD to detect afferent visual pathways involvement.

\section{Materials and methods}

We recruited nine Spanish patients with clinically and genetically confirmed SCA-3/MJD diagnosis (more than 52 CAG repeats) in the Hospital Clínic de Barcelona between January 2010 and January 2011. The protocol for this study was approved by the local hospital ethics committee, and informed consent was obtained from all patients. The study and data accumulation were performed in conformity with Spanish and European laws and in adherence to the tenets of the Declaration of Helsinki.

Neurological history, age at onset, and evolution of the disease were recorded in all patients. To assess the disease severity the Scale for the Assessment and Rating of Ataxia (SARA) was used. The SARA ranges from 0 (no ataxia) to 40 (most severe ataxia), being a result of the evaluation of eight quantitative features for gait, stance, sitting, speech disturbance, and limb kinetic functions. Each patient had a complete ophthalmologic examination, including best-corrected visual acuity (BCVA) with Snellen chart, colour vision (Ishihara's test), ocular motility, slit lamp biomicroscopy, intraocular pressure measurement, indirect ophthalmoscopy, and visual field test (Humphrey Systems GPA II, Carl-Zeiss Meditec, Dublin, CA, USA). Spectral-domain OCT (Cirrus HD software version 3.0.0.64, Carl-Zeiss Meditec) was performed in all patients to measure the RNFL thickness and the macular thickness. The OCT acquisition protocols adopted were Optic Disc Cube $200 \times 200$ and Macular Cube $521 \times 128$, which were subsequently analysed with the RNFL Thickness and Macular Thickness protocols. For each eye, we studied the mean RNFL thickness, temporal, superior, nasal and inferior quadrant thickness, and macular average thickness. Patients who returned in the follow-up were re-evaluated, OCT was repeated and a progression rate was calculated. Patients were excluded if they had another cause of visual loss, another cause of decrease in the RNFL thickness (glaucoma and other optic neuropathy), or maculopathies. Also patients with large refractive defects (hyperopia $>5$ diopters and myopia $>5$ diopters) were excluded because of the variability of RNFL thickness measurements in this population. RNFL thickness and macular thickness were correlated with the SARA score and years of evolution of the disease. The General Lineal Model (GLM) approach was used in order to evaluate the relation between RNFL and SARA score or the duration of the disease with univariate and multivariate models. Owing to the sample size, a bootstrap estimation from 1000 samples was used as final estimation of these slopes with their 95\% confidence intervals (95\% CI). SPSS ver18 for Windows (SPSS Inc., Chicago, IL, USA) was used for statistical calculations.

\section{Results}

Nine patients were included in the study, five females $(55.56 \%)$ and four males $(44.44 \%)$. Two families were recruited: patients 1,2, and 3 were brothers, and patients 4 and 5 were sisters. The mean age was 46.33 -year-old (standard deviation (SD) \pm 10 ), ranging from 35 to 63 years. The mean age at the onset of the disease was 36.33-year-old ( $\mathrm{SD} \pm 9.26$ ), ranging from 23 to 50 years. Mean disease duration was 10 years $(S D \pm 5,31)$, ranging from 1 to 17 years (see Table 1).

All patients presented gait ataxia at the moment of ophthalmic examination as well as dysarthria and dysmetria. Patients 1, 2, 4, and 8 had autonomous gait, patients 5 and 6 had difficulties with gait, and finally patients 3, 7, and 9 needed wheelchairs. Patients 4, 5, and 6 also presented spasticity. SARA score was determined in all of them (see Table 1). The mean SARA score was 14.67 , with a SD of \pm 5.78 , ranging from 5 to 22 .

At the last follow-up visit mean BCVA was 20/25, ranging from $20 / 30$ to $20 / 20$. Colour vision was preserved in all patients, but one who was previously reported of congenital dyscromatopsia. Visual field test exams were normal in all patients, with an average mean defect of 1.74 and mean SD of 1.86. All patients presented some degree of ocular motility alterations described in this condition such as gaze-evoked nystagmus or hypometric saccades. Fundus examination was normal in all patients.

The mean RNFL thickness was 77.39 microns, SD was \pm 5.93 . The mean RNFL thickness in the superior quadrant was 95.22 microns (SD \pm 12.90$)$, in the inferior 
Table 1 Epidemiology and neurological findings in our patients. Average values and SDs are reported

\begin{tabular}{llcccccc}
\hline Patients & Gender & Age (years) & Age at onset (years) & Disease duration (years) & Ataxia & Wheelchair bound & SARA Score \\
\hline 1 & Female & 49 & 40 & 9 & Yes & No & 9 \\
2 & Male & 35 & 34 & 1 & Yes & No & 5 \\
3 & Female & 47 & 30 & 17 & Yes & Yes & 17 \\
4 & Female & 37 & 30 & 7 & Yes & No & 9.5 \\
5 & Female & 40 & 30 & 10 & Yes & No & 18.5 \\
6 & Male & 36 & 23 & 16 & Yes & No & 18.5 \\
7 & Female & 63 & 49 & 14 & Yes & Yes & 22 \\
8 & Female & 56 & 51 & 15 & Yes & No & 13 \\
9 & Male & 54 & $36.3 \pm 9.26$ & $10 \pm 5.32$ & Yes & Yes & 19.5 \\
& Male 33.3\% & $46.3 \pm 10$ & & & & $33.3 \%$ & $14.67 \pm 5.78$ \\
& Female 66.6\% & & & & & \\
\hline
\end{tabular}

Table 2 OCT findings in our nine patients, including RNFL and macular thickness total values, average values, and SD

\begin{tabular}{|c|c|c|c|c|c|c|c|}
\hline Patient & Eye & $\begin{array}{c}\text { Mean RNFL } \\
\text { thickness }\end{array}$ & $\begin{array}{c}\text { Superior RNFL } \\
\text { thickness }\end{array}$ & $\begin{array}{c}\text { Inferior RNFL } \\
\text { thickness }\end{array}$ & $\begin{array}{c}\text { Temporal RNFL } \\
\text { thickness }\end{array}$ & $\begin{array}{c}\text { Nasal RNFL } \\
\text { thickness }\end{array}$ & $\begin{array}{c}\text { Mean macular } \\
\text { thickness }\end{array}$ \\
\hline 1 & OD & 89 & 107 & 111 & 59 & 81 & 259 \\
\hline 1 & OS & 87 & 114 & 101 & 51 & 83 & 258 \\
\hline 2 & OD & 80 & 89 & 93 & 56 & 51 & 266 \\
\hline 2 & OS & 76 & 98 & 81 & 58 & 45 & 266 \\
\hline 3 & OD & 79 & 93 & 97 & 51 & 74 & 260 \\
\hline 3 & OS & 79 & 100 & 92 & 49 & 77 & 262 \\
\hline 4 & OD & 79 & 100 & 96 & 60 & 61 & 280 \\
\hline 4 & OS & 80 & 108 & 101 & 57 & 54 & 277 \\
\hline 5 & OD & 79 & 90 & 100 & 66 & 60 & 250 \\
\hline 5 & OS & 73 & 87 & 88 & 58 & 59 & 258 \\
\hline 6 & OD & 76 & 81 & 99 & 65 & 59 & 249 \\
\hline 6 & OS & 72 & 99 & 93 & 52 & 45 & 247 \\
\hline 7 & OD & 74 & 91 & 91 & 53 & 61 & 212 \\
\hline 7 & OS & 73 & 90 & 91 & 56 & 55 & 206 \\
\hline 8 & OD & 75 & 100 & 85 & 48 & 65 & 223 \\
\hline 8 & OS & 86 & 120 & 103 & 55 & 62 & 221 \\
\hline 9 & OD & 66 & 65 & 92 & 55 & 52 & 276 \\
\hline 9 & OS & 70 & 82 & 87 & 58 & 46 & 277 \\
\hline Results & & $77.39 \pm 5.93$ & $95.22 \pm 12.91$ & $94.50 \pm 7.28$ & $55.94 \pm 4.91$ & $60.56 \pm 11.72$ & $252.61 \pm 22.80$ \\
\hline
\end{tabular}

Abbreviations: OCT, optical coherence tomography; RNFL, retinal nerve fibre layer.

94.50 (SD \pm 7.28$)$, temporal $55.94(\mathrm{SD} \pm 4.91)$, and nasal 60.56 (SD \pm 11.72 ) (see Table 2). In 15 eyes, (83.33\%) the mean RNFL thickness was lower than the population average considering age and sex. In 10 cases, there was a reduction of the RNFL thickness in the superior sector, 8 in the inferior and 4 in the nasal. Temporal sector RNFL thickness was preserved in all eyes.

The mean macular thickness was 252.61 microns, $\mathrm{SD} \pm 22.80$, being inferior respecting average population in only 2 of the 18 eyes of the study (11.11\%). All patients had integrity of photoreceptor inner/outer junction.

In four patients, (eight eyes) OCT studies were performed during a mean follow-up of 14.25 months (ranging from 9 to 24 months), and in five eyes (62.50\%) there was a mild trend to a RNFL thickness decrease in this period. A progression rate was calculated, resulting in a loss of $8,6,2$, and 2, and 1 average RNFL thickness per year (mean-3.60 per year, $\mathrm{SD} \pm 3.03$ ).
The univariate approach showed an inversely proportional correlation between the SARA score and the RNFL thickness, but not between disease duration and RNFL thickness (see Figure 1 and Table 3). On the other hand, macular thickness showed an inverse correlation with duration of the disease; however, results regarding the SARA score were not conclusive (see Table 3).

\section{Discussion}

RNFL alterations have been observed in the preclinical stage of dominant optic atrophy (DOA ${ }^{6}$ and Leber's hereditary optic neuropathy (LHON). ${ }^{7}$ Moreover, in some neurodegenerative diseases such as Parkinson's disease and Alzheimer's disease, the RNFL thinning has been demonstrated with OCT, even in patients without visual loss. ${ }^{4,5}$ Considering neurodegenerative ataxias, such as Friedreich's ataxia, alterations in the afferent 


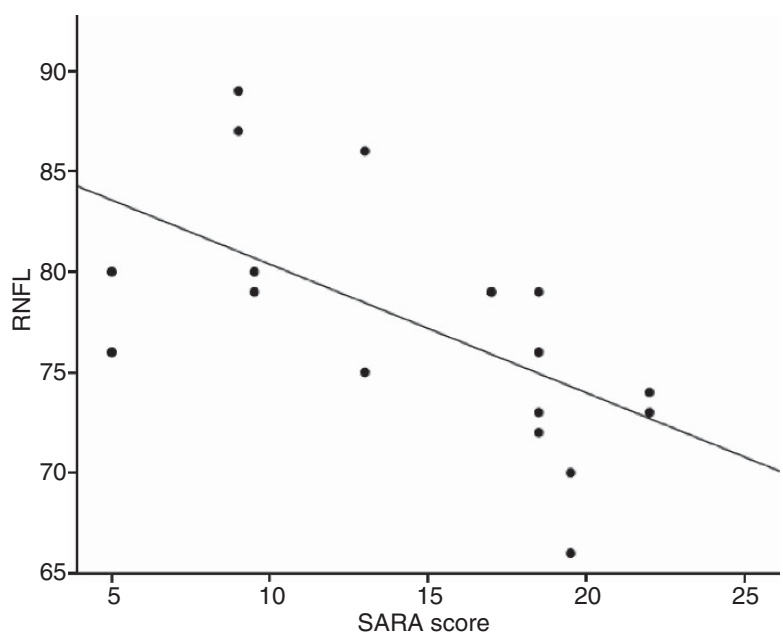

Figure 1 Linear negative correlation between SARA score and RNFL thickness is found in the univariate approach.

Table 3 Statistical analysis with the univariate approach between RNFL thickness and duration of the disease (no significant correlation), and SARA score (significant negative correlation)

\begin{tabular}{llcc}
\hline RNFL & Parameter & Slope and 95\% CI & P-value \\
\hline $\begin{array}{l}\text { Univariate } \\
\text { approach }\end{array}$ & $\begin{array}{l}\text { Duration of } \\
\text { disease (years })\end{array}$ & $0.15(-0.35 ; 0.68)$ & 0.537 \\
& SARA score & $-0.64(-1.16 ;-0.29)$ & 0.012
\end{tabular}

Abbreviations: RNFL, retinal nerve fibre layer; SARA, Scale for the Assessment and Rating of Ataxia.

visual pathway, expressed by a reduction of the RNFL thickness in the OCT can be ascertained. ${ }^{3}$ However, in the MJD, and other SCA, despite some results that have been recently published, there are no conclusive studies regarding RNFL and macular thickness variations (except SCA-7 with its cone and rod macular dystrophy associated). For example, Stricker $e a^{8} l^{8}$ found a decreased average RNFL thickness in a group of SCA-1 patients compared with age and sex matched healthy control group, mainly affecting the temporal sector. In addition, seven of the nine patients evaluated presented at least one sector below the normal range. However, in their study of OCT findings in SCA-1, 2, 3, and Cerebellar Multisystem Atrophy (CMA), Pula et $a l^{9}$ described a RNFL thinning in SCA-2 and SCA-3 but not in SCA-1. Therefore, results in SCA-1 are controversial. Regarding OCT findings in SCA-3, Pula et $a l^{9}$ studied five patients (10 eyes) and found an overall RNFL thickness decrease compared with a control group, but the authors failed in analysing which sectors of the RNFL were affected. Moreover, although compared with the average RNFL with a control group, it was not specified which cases were below the normality in the individual analysis performed by the OCT device. In our study, we found a mean average RNFL thickness of 77.39 microns, with a mild decrease in 15 of 18 eyes considering the analysis of the OCT, that represented the $83.33 \%$ of the studied eyes. Thus, SCA-3 patients can present a subclinical RNFL loss not attributable to any ophthalmic disease, and then probably secondary to their neurodegenerative illness. There was not a specific pattern in these cases with RNFL loss, being affected the superior, inferior, and nasal sectors in 10, 8 , and 4 eyes, respectively. Temporal sector was preserved in all of the studied eyes; therefore, papillomacular bundle probably is not primarily involved in SCA-3 and consequently visual acuity is preserved in these patients (mean BCVA 20/25 and colour vision conserved in all patients, except the one affected of congenital dyscromatopsia). For instance, RNFL thinning observed in our study showed predominance for the superior and inferior sectors, which could mimic glaucomatous optic disc neuropathy, thus an exhaustive ophthalmologic examination, including intraocular pressure measurement and visual field testing, should be performed in these patients. In that sense, our results differ substantially of the ones published by Stricker $e t a l,{ }^{8}$ who reported a temporal fibre affection in SCA-1, resembling pathology found in toxic or mitochondrial optic nerve diseases. Thus, the physiopathology of the neuro-opthalmologic degeneration in SCA-3 seems to be more similar to the observed in Alzheimer's and Parkinson's disease rather than in the SCA-1 and toxic or mitochondrial optic nerve diseases. Interestingly, although being classified in the same group of dominant inherited ataxias, our results and the previous reported show that SCA are a heterogeneous group of ataxias, each one with its own physiopathology. However, it should be noted that different OCT devices had been used in the studies mentioned, and therefore results cannot be directly compared.

A macular thickness decrease has been observed in dominant optic atrophy (DOA), due to the degeneration of retinal ganglion cells (RGC) of the papillomacular bundle, which is thought to subsequently produce the RNFL loss. ${ }^{10}$ On the other hand, the cause of RNFL loss in Alzheimer's and Parkinson's disease is multifactorial, including degeneration of RGC and through retrograde degeneration due to the loss of cortical neurons. $., 11,12$ Pula $e t a l^{9}$ found a statistical significant decrease of mean macular thickness in five patients affected of SCA-3 compared with a control group. The thinning was significant in nasal and inferior sectors of the macula, probably secondary to a RGC loss of the papillomacular bundle in these sectors. However, in our study the mean macular thickness was 252.61 microns, and only in 2 of the 18 eyes $(11.11 \%)$ a macular thickness reduction was 
observed. Therefore, considering our results, the cause of the RNFL loss observed in our patients seems to probably be multifactorial, and could be explained as a result of a retrograde degeneration, similarly as in Alzheimer's disease, instead of being secondary to a RGC loss. On the other hand, in some SCAs a cone-rod dystrophy has been described as a part of the disease, but neither the fundus examination nor the SD-OCT demonstrated any evidence of a photoreceptor dysfunction. The macular SD-OCT showed an integrity of the photoreceptor inner/outer junction in all cases. Moreover, the preservation of visual function (visual acuity, colour vision, and visual field test) should be noted favourably to our hypothesis. However, it should be noted that electrophysiological studies and the use of low contrast visual acuity charts would be more sensitive in order to detect subclinical visual dysfunction, and therefore should be used in future studies.

The median SARA score was 17 and ranged from 5 to 22 in our patients. Considering the range of this scale (from 0 to 40) none of our patients had a very severe ataxia, for instance this could explain that the RNFL thickness decrease detected in our study was mild. A statistically significant negative correlation was found between SARA score and RNFL thickness with the univariate model. Stricker $e a^{8}$ did not found significant level for the correlation. Probably due to small samples of both studies, a statistically significant correlation is difficult to be reached, but in our study was found, and it is consistent with the fact that the most clinically affected patients should have a less RNFL thickness. Therefore, our study shows that a clinical scale and an anatomic measurement are correlated, thus RNFL thickness could be considered as a promising biomarker and a complementary tool in a severity grading scale of the SCA-3.

The mean evolution of the SCA was 10 years, ranging from 1 to 17 years. Interestingly, a statistically significant negative correlation with duration of the disease and RNFL thickness was not found with the univariate approach. A possible explanation of this result could be the lack of statistical power due to the small and heterogeneous (SD of 5.32 regarding years of evolution) sample of our study. However, SCA-3 mutation is an expanded CAG repeat that encodes polyQ in the disease gene product ataxin-3, which means that the phenotype in MJD varies widely depending on repeat length. ${ }^{13}$ Thus, a RNFL loss could be more probably detected in the most severe forms with more CAG repeats and not in all patients despite the years of the disease evolution. Furthermore, these patients should have also the most intense RNFL loss. For instance, we should also consider other variables besides time of evolution of the disease such as number of CAG repeats. In addition, probably the severity of the disease could be more related with the severity of the mutation rather than the time of evolution. In our study, despite the fact that all patients were genetically confirmed for SCA-3, unfortunately the exact number of CAG copies was available in only three patients. Patient 2 had 70 copies, patient 4 had 71 copies, and patient 9 had 71 copies. Further studies including this issue need to be performed in order to confirm our hypothesis.

As an alternative method, we considered the age at the diagnosis of the disease; then, we should expect that youngest patients, in the moment of the diagnosis, have a greater number of CAG repeats and a more severe form of the disease, so they could have more neuronal loss than older ones with a similar time of evolution. Therefore, we analysed whether there was a correlation between age at the neurological diagnosis and age at the moment of the ophthalmologic examination, and RFNL and macular thickness. Again, the small size and heterogeneity of our studied population (youngest patient was 23 and oldest 50 years, with a SD of 9.26 years) did not permit to find any statistically significant differences.

An OCT study during a mean follow-up of 14.25 months (ranging from 9 to 24 months) was performed in four patients (eight eyes) to assess the evolution of the RNFL. Five of the eight eyes $(62.50 \%)$ showed a mild trend of RNFL reduction ( -3.60 per year, SD \pm 3.03 ) during this period, the other three remained stable and in one case gained 2 microns. Considering that it is unlikely to gain RNFL thickness this case should be considered as a result of the variability between the exams. Also the three cases with a RNFL loss of $-2,-2$, and -1 mean RNFL thickness could be considered as well. However, the two cases with a RNFL loss of -8 and -6 microns per year should be noted as a possible progression. In a study that evaluated the reproducibility of the Cirrus HD Spectral-Domain OCT in multiple sclerosis patients, Syc et al ${ }^{14}$ found excellent results even in inter-rater exams. Moreover, in the detection and assessment of RNFL progression in glaucoma, spectral-domain OCT has been superiorly proven to time-domain OCT. ${ }^{15}$ In that sense, although OCT Cirrus HD is an excellent tool to detect progression, because of the small sample of the study and the absence of a control group of normal subjects, it is not possible to assure that this loss detected in our study represents a progression of the disease and it is not attributable to other causes such as variability between the exams.

\section{Conclusions}

SCA-3 is an uncommon neurodegenerative disease of the cerebellum and its connections, with a variable phenotype due to the characteristics of the gene mutation (a CAG expansion). A mild decrease of the RNFL 
thickness in most eyes (83.33\%) was found in our study. RNFL thickness was negatively correlated with the SARA score but not with the duration of the disease in the univariate models. Therefore, RNFL thickness could be used as a biomarker and clinically severity grading system of the disease. On the other hand, none of the univariate approaches showed correlation between macular thickness and SARA score or years of evolution of the ataxia. However, considering the low prevalence of the disease and its heterogeneity, multicentric studies should be performed in order to confirm our initial results.

\section{Summary}

What was known before

- Retinal nerve fibre layer could be affected in some neurodegenerative ataxias (including some types of spinocerebellar ataxia such as SCA-1).

\section{What this study adds}

- Decrease in the retinal nerve fibre layer in Spanish patients affected of SCA-3.

- Negative correlation between retinal nerve fibre layer thickness and SARA score.

- Preservation of temporal sector and macular thickness.

\section{Conflict of interest}

The authors declare no conflict of interest.

\section{References}

1 Paulson HL. The spinocerebellar ataxias. J Neuro-Ophthalmol 2009; 29(3): 227-237.

2 Miller RC, Tewari A, Miller JA, Garbern J, Van Stavern GP. Neuro-ophthalmologic features of spinocerebellar ataxia type 7. J Neuro-Ophthalmol 2009; 29(3): 180-186.

3 Fortuna F, Barboni P, Liguori R, Valentino ML, Savini G, Gellera $\mathrm{C}$ et al. Visual system involvement in patients with Friedreichs ataxia. Brain 2009; 132: 116-123.
4 Moschos MM, Tagaris G, Markopoulos I, Margetis I, Tsapakis S, Kanakis $\mathrm{M}$ et al. Morphologic changes and retinal impairment in patients with Parkinson disease without visual loss. Eur J Ophthalmol 2010; 21: 24-29.

5 Lu Y, Li Z, Zhang X, Ming B, Jia J, Wang R et al. Retinal nerve fibre layer structure abnormalities in early Alzheimers disease: evidence in optical coherence tomography. Neurosci Lett 2010; 480: 69-72.

6 Kim TW, Hwang JM. Stratus OCT in dominant optic atrophy. J Glaucoma 2007; 16: 855-858.

7 Barboni P, Carbonelli M, Savini G, Ramos Cdo V, Carta A, Berezovsky A et al. Natural history of Lebers hereditary optic neuropathy: longitudinal analysis of the retinal nerve fibre layer by optical coherence tomography. Ophthalmology 2010; 117: 623-627.

8 Stricker S, Oberwahrenbrock T, Zimmermann H, Schroeter J, Endres M, Brandt AU et al. Temporal retinal nerve fibre loss in patients with spinocerebellar ataxia type 1. PLoS One 2011; 6(7): e23024.

9 Pula JH, Towle VL, Staszak VM, Cao D, Bernard JT, Gomez CM et al. Retinal nerve fibre layer and macular thinning in spinocerebellar ataxia and cerebellar multisystemic atrophy. Neurophthalmology 2011; 35(3): $108-114$.

10 Ito $\mathrm{Y}, \mathrm{Nakamura}$ M, Yamakoshi T, Lin J, Yatsuya H, Terasaki H. Reduction of inner retinal thickness in patients with autosomal dominant optic atrophy associated with OPA-1 mutations. Invest Ophthalmol Vis Sci 2007; 48: 4079-4086.

11 Hinton DR, Sadun AA, Blanks JC, Miller CA. Optic nerve degeneration in Alzheimers disease. N Engl J Med 1986; 315: 485-487.

12 Djamgoz MB, Hankins MW, Hirano J, Archer SN. Neurobiology of retinal dopamine in relation to degenerative states of the tissue. Vision Res 1997; 37: 3509-3529.

13 Soong BW, Paulson HL. Spinocerebellar ataxias: an update. Curr Opin Neurol 2007; 20: 438-446.

14 Syc SB, Warner CV, Hiremath GS, Farrell SK, Ratchford JN, Conger A et al. Reproducibility of high-resolution optical coherence tomography in multiple sclerosis. Mult Scler 2010; 16(7): 829-839.

15 Leung CK, Chiu V, Weinreb RN, Liu S, Ye C, Yu M et al. Evaluation of retinal nerve fiber layer progression in glaucoma. A comparison between spectral-domain and time-domain optical coherence tomography. Ophthalmology 2011; 118(8): 1558-1562. 